\title{
Complexions at the Electrolyte/Electrode Interface in Solid Oxide Cells
}

Hanna Türk ${ }^{\mathrm{a}, \mathrm{b}, \dagger}$, Franz-Philipp Schmidtt, ${ }^{\mathrm{c}, \dagger}$, Thomas Götsch ${ }^{\mathrm{c}, \dagger},{ }^{*}$, Frank Girgsdies $^{c}$, Adnan Hammud ${ }^{c}$, Danail Ivanov ${ }^{c}$, Izaak C. Vinke ${ }^{e}$, L.G.J. (Bert) de Haart $^{\mathrm{e}}$, Rüdiger-A. Eichel ${ }^{\mathrm{e}, \mathrm{f}}$, Karsten Reuter ${ }^{\mathrm{a}, \mathrm{b}}$, Robert Schlögl ${ }^{\mathrm{c}, \mathrm{d}}$, Axel Knop-Gericke ${ }^{\mathrm{c}, \mathrm{d}}$, Christoph Scheurer ${ }^{\mathrm{a}, \mathrm{b}}$, and Thomas Lunkenbein ${ }^{\mathrm{c}}$

aTechnische Universität München, Department of Chemistry, Chair for Theoretical Chemistry and Catalysis Research Center, 85748 Garching, Germany

${ }^{\text {b} F r i t z-H a b e r-I n s t i t u t ~ d e r ~ M a x-P l a n c k-G e s e l l s c h a f t, ~ T h e o r y ~ D e p a r t m e n t, ~} 14195$ Berlin, Germany

${ }^{\mathrm{c}}$ Fritz-Haber-Institut der Max-Planck-Gesellschaft, Department of Inorganic Chemistry, 14195 Berlin, Germany

dMax Planck Institute for Chemical Energy Conversion, Department of Heterogeneous Reactions, 45470 Mülheim an der Ruhr, Germany

érschungszentrum Jülich GmbH, Institute of Energy and Climate Research, Fundamental Electrochemistry (IEK-9), 52425 Jülich, Germany

${ }^{\mathrm{f}}$ RWTH Aachen University, Institute of Physical Chemistry, 52056 Aachen, Germany

${ }^{\dagger}$ These authors have contributed equally to this work.

*E-Mail: goetsch@fhi-berlin.mpg.de 
Rapid deactivation presently limits a wide spread use of high-temperature solid oxide cells (SOCs) as otherwise highly efficient chemical energy converters. With deactivation triggered by the ongoing conversion reactions, an atomic-scale understanding of the active triple-phase boundary (TPB) between electrolyte, electrode and gas phase is essential to increase cell performance. Here we use a multi-method approach comprising transmission electron microscopy and first-principles calculations and molecular simulations to untangle the atomic arrangement of the prototypical SOC interface between a lanthanum strontium manganite (LSM) anode and an yttria-stabilized zirconia (YSZ) electrolyte. We identify an interlayer of selflimited width with partial amorphization and strong compositional gradient, thus exhibiting the characteristics of a complexion that is stabilized by the confinement between two bulk phases. This offers a new perspective to understand the function of SOCs at the atomic scale. Moreover, it opens up a hitherto unrealized design space to tune the conversion efficiency. 


\section{Introduction}

The intermittent output of renewable energy sources such as wind or solar power requires an efficient way for the mid-term storage of excess electrical energy. Solid oxide fuel and electrolysis cells (SOFCs, SOECs) offer the prospect of high performance devices, ${ }^{[1,2]}$ reaching more than $80 \%$ efficiency, ${ }^{[3]}$ while, at the same time, providing mid- to long-term chemical storage capability.

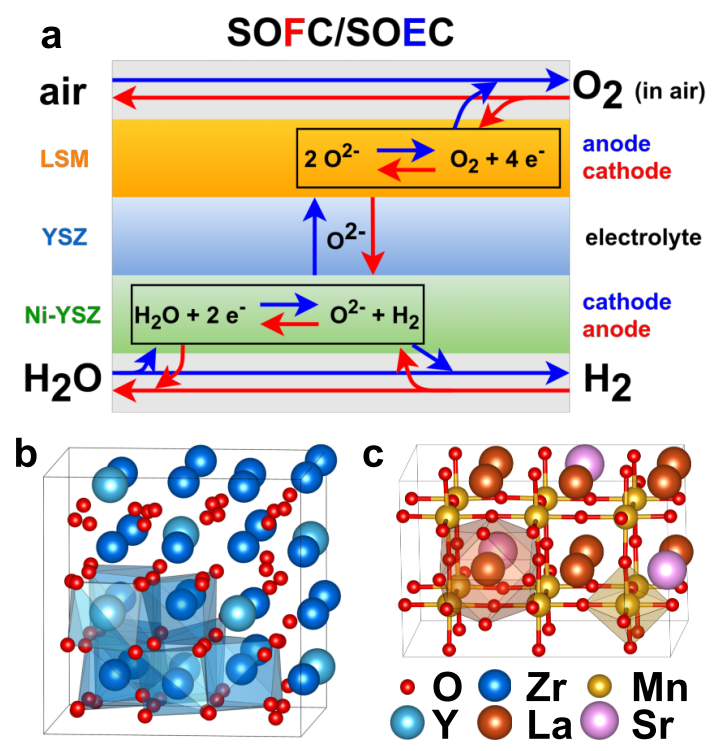

Figure 1. a) Schematic representation of the bifunctionality of solid oxide cells. The reaction pathways for fuel cell (red) and electrolysis operation mode (blue) are indicated by different colors. Unit cells of b) 10YSZ (YSZ with $\left.10 \mathrm{~mol} \% \mathrm{Y}_{2} \mathrm{O}_{3},\left(\mathrm{Y}_{2} \mathrm{O}_{3}\right)_{0.1}\left(\mathrm{ZrO}_{2}\right)_{0.9}\right)$ and c) LSM25 (LSM with 25 at\% of $\mathrm{Sr}$ in the cuboctahedral A site of the general $\mathrm{ABO}_{3}$ perovskite, $\mathrm{La}_{0.75} \mathrm{Sr}_{0.25} \mathrm{MnO}_{3}$ ) for one probable doping distribution, relaxed using density functional theory (DFT). The deviations from the experimentally used concentrations are necessary to obtain cell sizes suitable for DFT. The elements are labelled by color and atomic-radii-dependent spheres as defined in the legend. Polyhedra were added to each structure indicating coordination environments.

As shown in Figure 1a, these cells typically consist of an oxygen-ion-conducting ceramic electrolyte such as yttria-stabilized zirconia (YSZ, $\left.\left(\mathrm{Y}_{2} \mathrm{O}_{3}\right)_{x}\left(\mathrm{ZrO}_{2}\right)_{1-x}\right)$ and a Ni/YSZ fuel electrode. A perovskite like lanthanum strontium manganite (LSM, (La,Sr) $\mathrm{MnO}_{3-\delta}$ ) acts as air electrode where it is typically mixed with YSZ. ${ }^{[3-5]}$ The electrolyzer cell converts electrical to chemical energy e.g. by splitting water into $\mathrm{H}_{2}$ and $\mathrm{O}_{2}$, which can be stored. The reversed 
operation in fuel cell mode enables the back-transformation of chemical energy to electricity by oxidizing $\mathrm{H}_{2}$ with $\mathrm{O}_{2}$ to form $\mathrm{H}_{2} \mathrm{O}$. This bifunctionality of solid oxide cells is depicted in Figure 1a. The key to the high efficiency of these cells is the elevated operation temperature, which typically exceeds $1000 \mathrm{~K}$. However, commercial operation is not yet practical as the cells suffer from degradation even under steady state conditions. ${ }^{[6]}$ This needs to be mitigated to render a widespread adoption of SOECs and SOFCs feasible and overcome intermittent power availability. ${ }^{[7]}$ Predominant degradation occurs at the anode of the electrolyzer cells, where the oxygen evolution reaction (OER) shows a higher kinetic limitation compared to the processes taking place at the cathode. ${ }^{[8,9]}$

For a purely electron-conducting air electrode such as LSM, the triple phase boundary (TPB) between YSZ, gas phase and LSM is considered to be the active site. ${ }^{[4]}$ In this assembly, YSZ provides $\mathrm{O}^{2-}$, whereas the formed molecular oxygen will escape to the gas phase and LSM is used for current collection. Thus, an understanding of the degradation processes can be achieved by investigating the morphological and chemical changes of the TPB, such as reduction of the number of TPBs by delamination, or chemical reactions at the electrolyte/electrode interface. Even though the interfacial region has been subject to investigations before, ${ }^{[10-17]}$ many questions that are crucial for the overall cell performance concerning interfacial properties, microstructure and stability are still unanswered. A lack of theoretical support for a comprehensive structural analysis has rendered the detailed structure at the interface of YSZ with an LSM grain underneath the TPB, and even more so the TPB's structure itself, elusive. The assumption of an idealized, atomically thin, truly two-dimensional (2D) interface region makes the TPB a one-dimensional line with minimal active surface. Additionally, the transport of oxygen ions from YSZ to the catalytically active LSM, as well as the elementary steps of the reaction at the TPB, including oxygen exchange with the gas phase, remain unclear when considering neighboring pure LSM and YSZ phases with bulk properties. ${ }^{[18-20]}$ From detailed studies of intergranular interface regions in alloys and functional ceramic materials, a more diverse picture has emerged over the last decade, which comprises a variety of interface types. These range systematically from single layer, highly ordered interfaces, via double, triple, and multiple layer types with high residual order to fully disordered intergranular films (IGF) with a truly amorphous interphase between neighboring grains. The interfacial structural types in this classification system have been termed complexions. ${ }^{[21-25]}$ Complexions are not stable as a bulk three-dimensional (3D) phase. They can exist, though, as thermodynamically stable, quasi 
2D interfacial structures of finite, self-limiting width between adjacent bulk phases and exhibit reproducibly distinct physical and chemical properties. A surface cutting through a YSZ || complexion || LSM region or evolving like a spandrel from such an underlying complexion could present a TPB of finite width with mixed ionic/electronic conductivity (MIEC), explaining the propensity for oxygen exchange with the gas phase.

In the first part of this study, we use transmission electron microscopy (TEM) on the experimental side, and density functional theory (DFT) on the theoretical side to characterize the structural properties of the SOEC anode material and electrolyte at the nano and atomic scale. For this purpose, a half cell consisting of an LSM + YSZ air electrode, which reflects the anode of the electrolyzer, on top of an $8 \mathrm{YSZ}\left(\mathrm{ZrO}_{2}\right.$ with $\left.8 \mathrm{~mol} \% \mathrm{Y}_{2} \mathrm{O}_{3}\right)$ electrolyte is investigated. As current collector, pure LSM20 $\left(\left(\mathrm{La}_{0.8} \mathrm{Sr}_{0.2}\right)_{0.95} \mathrm{MnO}_{3-\delta}\right)$ is used. The results are interpreted with the aid of DFT calculations, allowing to establish a detailed picture of the electronic structure and state of the materials (see Figure $1 b$ and $c$ for the cubic crystal structures as obtained by theory). Based on the understanding gained from the material characterization, the interface of LSM and YSZ is examined in the final part using energy-dispersive X-ray spectroscopy (EDX), giving insights into the composition of the active phase, supported by force-field-based MC simulations. These, together with high-resolution scanning TEM (HR-STEM), are used to elucidate the structural configuration of the formed complexion at the atomic level.

\section{Results and Discussion}

\subsection{Crystallography of LSM}

As evidenced by X-ray diffraction (XRD) data shown in Figure S1 and S2 in the Supporting Information, LSM crystallizes in the rhombohedral perovskite structure $\left(\mathrm{ABO}_{3}\right)$ at room temperature. However, under operation conditions (i.e. 1000-1200 K), this structure has been shown to become pseudocubic, ${ }^{[26,27]}$ and at the typical sintering temperature of around $1400 \mathrm{~K}$, a fully cubic perovskite is formed. ${ }^{[28]}$ The rhombohedral structure can be conceived as a distorted cubic lattice where the $\left[\mathrm{BO}_{6}\right]$ octahedra are tilted around the 3 -fold rotation axis. ${ }^{[29]}$ This results in a displacement of the oxygen sites while the positions of the cations are unaffected. The structure is further analyzed in Figure 2. In the rhombohedral $\mathrm{rh}[241]$ viewing direction, which is equivalent to c[001] for the cubic perovskite, the oxygen atoms between the La sites split from single columns into two distinct locations. This is indicated by the red oxygen atoms 

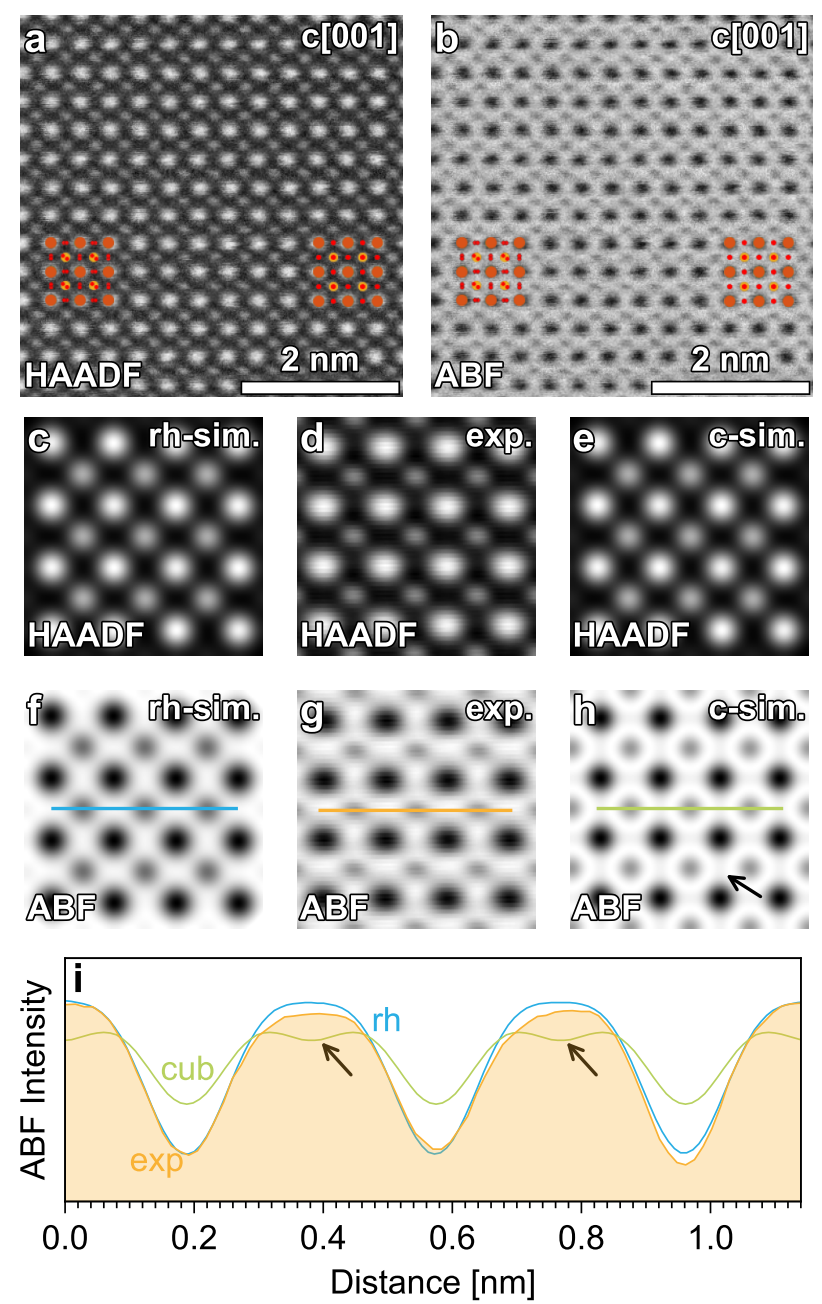

Figure 2. Bulk HAADF (a) and ABF (b) HR-STEM images of LSM in c[001] zone axis (corresponding to $\mathrm{rh}[241]$ ). The crystal structures of rhombohedral (left) and cubic (right) perovskites are overlaid on the images (La: orange, Mn: yellow, O: red), see Figure S3 in the Supporting Information. Experimental average over 1034 frames in a $14 \mathrm{~nm} \times 14 \mathrm{~nm}$ region by template matching $(\mathrm{d}, \mathrm{g})$ in comparison to simulated images for the rhombohedral $(\mathrm{c}, \mathrm{f})$ and cubic $(\mathrm{e}, \mathrm{h})$ perovskite. i) The experimental line profile (in c[100] direction across the Mn sites) of the ABF-STEM image agrees very well with the simulated intensity based on the rhombohedral structure. The dip due to the $\mathrm{O}$ atoms (black arrows), which is visible in the cubic case only, cannot be observed.

in the structural insets of Figure 2a and b (left inset: rhombohedral, right inset: cubic), as well as in Figure S3 in the Supporting Information in more detail. However, while the raw 
high-angle annular dark field STEM (HAADF-STEM) and annular bright field STEM (ABFSTEM) images in Figure 2a and b (most intense atoms: La, less intense: Mn), respectively, are generally adequate for investigating potential cation displacements and distortions, they exhibit an insufficient signal-to-noise ratio for determining the positions of the $\mathrm{O}$ sites. Thus, an average over 1034 unit cells within each image $(14 \mathrm{~nm} \times 14 \mathrm{~nm})$ was formed by template matching and rigid registration (see Figure S4 in the Supporting Information for more details). The results of this procedure are given in Figure 2d (HAADF-STEM) and 2g (ABF-STEM). For comparison, simulated STEM images with matching beam and microscope aberration parameters (see the Methods section) are displayed in Figure 2c and $2 \mathrm{f}$ (rhombohedral), as well as Figure $2 \mathrm{e}$ and $2 \mathrm{~h}$ (cubic). The cubic ABF-STEM image simulation (Figure $2 \mathrm{~h}$ ) hints at some contrast stemming from the $\mathrm{O}$ columns (black arrow), which is not the case for the rhombohedral ABF-STEM image (Figure 2f). The experimental average in Figure $2 \mathrm{~g}$ resembles more the rhombohedral simulation compared to the cubic simulation. This is corroborated by the horizontal line scans along the [100] direction through the Mn sites in Figure 2i. Whereas the cubic case (green line) shows a clear dip at the expected positions of the $\mathrm{O}$ atoms (marked by arrows), the rhombohedral simulation (blue) and the experimental intensity profile (orange shaded region) do not. This points towards a rhombohedral rather than a cubic structure for LSM. Although this averaging procedure might hide local contributions of single cubic unit cells inside a matrix of rhombohedral cells, this approach serves as a good way of determining the crystallographic phase on the nanometer scale from HR-STEM images. For instance, it has been reported that oxygen vacancies, which might be formed during OER operation, stabilize cubic phases of perovskites. ${ }^{[30]}$ Thus, while the resulting local crystallographic deviations would not be visible in bulk XRD data, the HR-STEM unit cell averaging approach would excel.

\subsection{The YSZ/LSM Interface}

The electrolyte/electrode interface constitutes an integral part within SOECs and SOFCs and its composition may influence the structure at the adjacent TPB and thus the performance. An in-depth view on the elemental spread across the YSZ/LSM interface is therefore crucial. In the following, the focus is put on the YSZ/LSM interface, which was investigated by a combined approach involving EDX, MC and HR-STEM. 

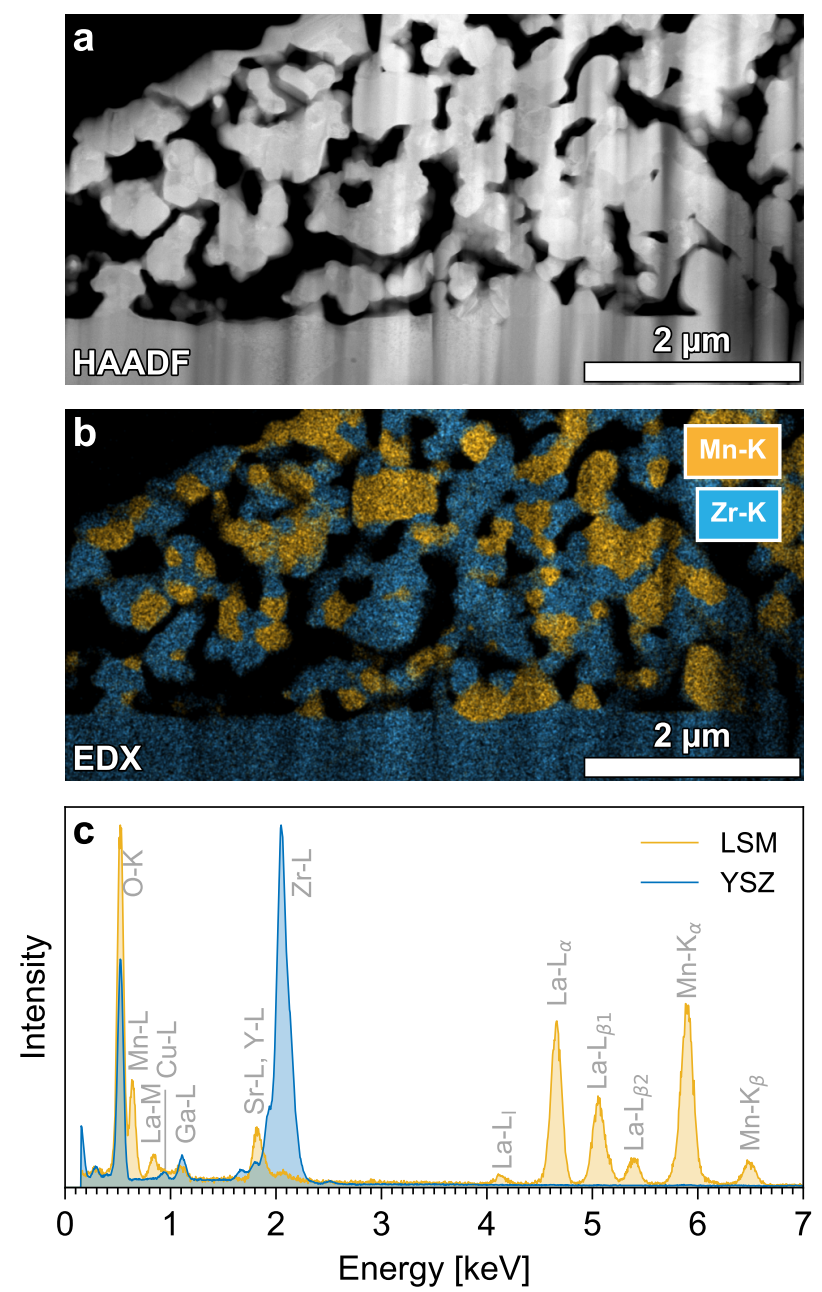

Figure 3. Morphology and elemental distribution in the screen-printed half cell. a) HAADFSTEM image of the interface between the 8YSZ electrolyte and the LSM + 8YSZ air electrode. b) Overlaid Mn-K and Zr-K intensity maps (EDX) from the region shown in (a). c) EDX spectra sampled from YSZ and LSM regions.

\subsubsection{Inter-Diffusion at the YSZ/LSM Interface}

Figure 3a displays an overview HAADF-STEM image recorded on a cross-sectional lamella of the electrolyte/electrode interface, prepared by a focused ion beam (FIB) inside an SEM. Simultaneously to the HAADF-STEM image, an EDX spectrum image was acquired. The elemental Mn and Zr distributions (based on the Mn-K and Zr-K line intensities) are shown in Figure 3b. The EDX maps at low magnification clearly show well separated phases in the size range of approximately $0.5-1 \mu \mathrm{m}$, which are highlighted by the blue $(\mathrm{Zr})$ and yellow $(\mathrm{Mn})$ colors in Figure 3b. The respective spectra for the Zr-rich and Mn-rich phases are displayed in Figure 3c using the same color code as in the elemental distribution map in Figure 3b. The EDX 
spectra show signals of all expected elements that are present in LSM and YSZ. Intensity that stems from the $\mathrm{Cu}-\mathrm{L}$ line arises from the copper lift-out grid on which the lamella is mounted. The Ga-L line is an artifact that stems from the FIB sample preparation.
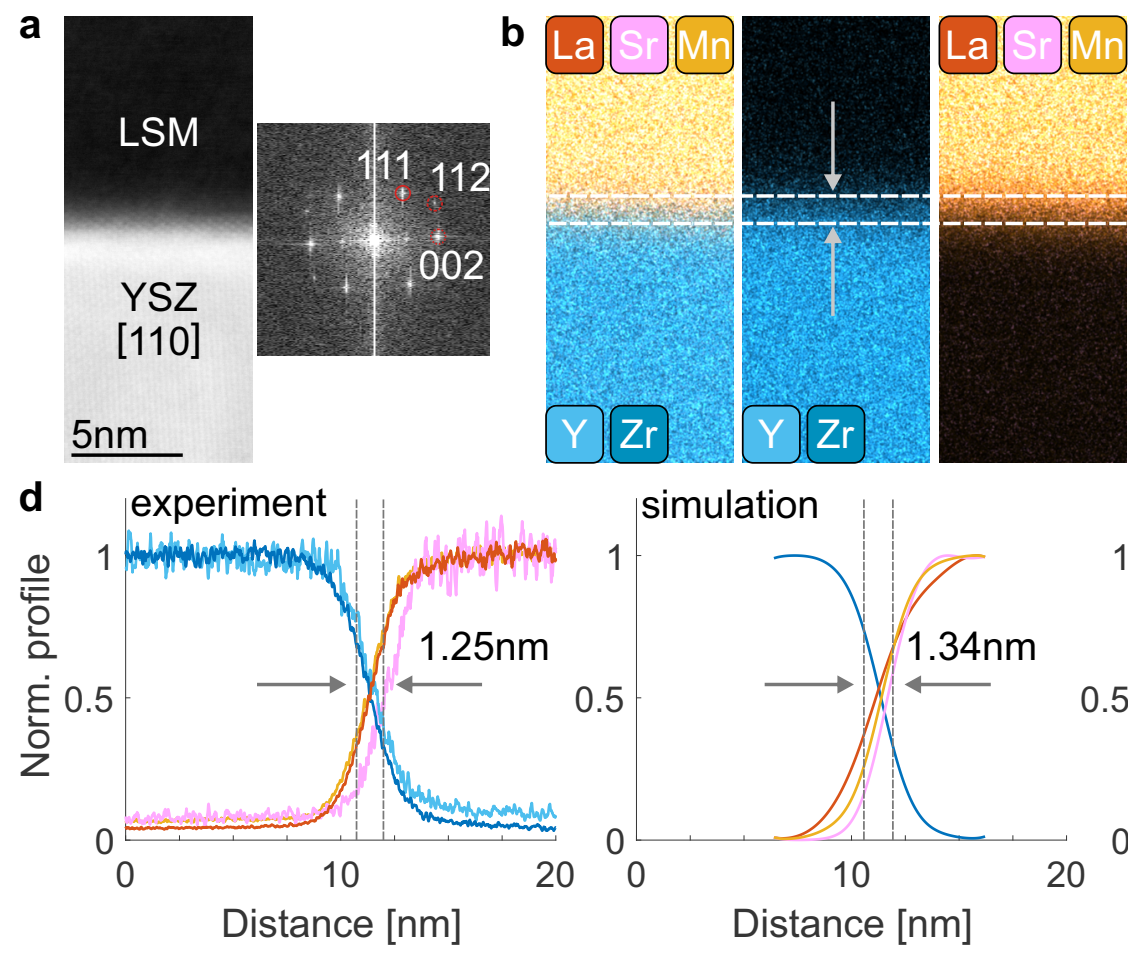

\begin{tabular}{|c|c|c|}
\hline & $\sigma_{\exp }[\mathrm{nm}]$ & $\sigma_{\text {sim }}[\mathrm{nm}]$ \\
\hline $\mathrm{Y}$ & 1.22 & --- \\
\hline $\mathrm{Zr}$ & 1.34 & 1.26 \\
\hline $\mathrm{La}$ & 1.26 & 1.74 \\
\hline $\mathrm{Sr}$ & 1.15 & 1.10 \\
\hline $\mathrm{Mn}$ & 1.26 & 1.26 \\
\hline \hline$\varnothing$ & 1.25 & 1.34 \\
\hline
\end{tabular}
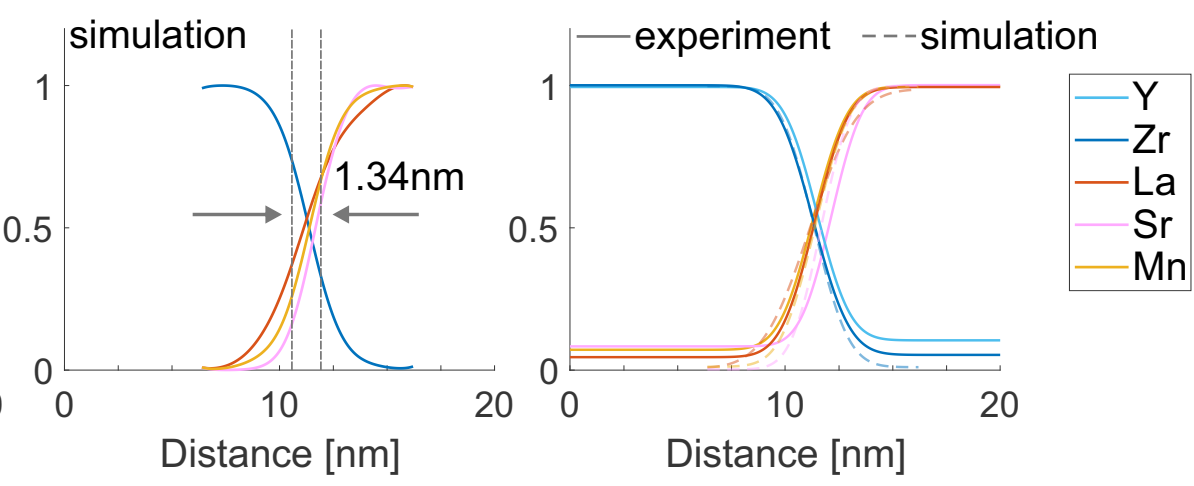

Figure 4. Inter-diffusion at the YSZ/LSM interface: a) DF-STEM image of YSZ/LSM interface, with YSZ oriented in a [110] zone axis (left), and corresponding Fourier-transformed image with reflexes highlighted by red circles, used for determining the [110] orientation of YSZ (right). b) EDX maps of elements as indicated by the insets, with LSM on top of YSZ. The dashed lines highlight the mixed LSM + YSZ inter-diffusion region. c) Experimental and simulated elemental spread across the YSZ/LSM interface. d) Experimental line profiles extracted from the EDX maps in (b) (left), simulated line profiles (middle), and comparison of fits (right) from experiment (solid) and simulation (dashed). The indicated values of $1.25 \mathrm{~nm}$ (experiment) and $1.34 \mathrm{~nm}$ (simulation) are the arithmetic mean of the values given in (c), with sigma being the standard deviation of the normal distribution function. Note the significant shift of Sr of approximately $0.8 \mathrm{~nm}$ with respect to all other elements.

The results from a high magnification EDX study are provided by Figure 4: Figure 4a shows a dark field STEM (DF-STEM) electron micrograph in which the LSM phase is on top of YSZ (see Figure S11 and Figure S12 in the Supporting Information). The latter is oriented along the [110] axis in viewing direction, which results in Laue reflections in the Fourier plane, identified 
as 111, 112 and 002 (Figure 4a, red circles in the corresponding FFT image). ${ }^{[31]}$ The elemental maps shown in Figure 4b include La, Sr and Mn (reddish colors) and Y and Zr (blueish colors), in superimposed (left) and separated (middle and right) representation. At the grain boundary, elements from LSM intermix with elements from YSZ and an extended compositionally unique region (as indicated by white dashed lines and grey arrows, Figure 4b). These experimental findings were corroborated by MC simulations (see the Methods section for details). Values for the width of the intergranular layer derived from experiment and simulation are presented in Figure 4c. They agree very well except for La, which is discussed below. Further note that, in the case of $\mathrm{Y}$, the amount of doping is too small for the size-limited simulation cell to obtain a fully reliable line profile. Supplementary details on the EDX quantification are presented in Figure S13 and S14 of the Supporting Information for experiment and simulation, respectively.

A more detailed view on the elemental distribution across the interface is given in Figure 4d by the corresponding elemental line profiles: the left panel shows the EDX signal across the interface for La, Sr, Mn, Y and Zr. From these line profiles, an average inter-diffusion width of $1.25 \mathrm{~nm}$ was identified, indicated by grey arrows and the vertical lines. The middle panel in Figure $4 \mathrm{~d}$ shows the simulated results, which, with an $1.34 \mathrm{~nm}$ average diffusion layer, are again qualitatively close to the experimental curves. As opposed to the experiment, a broadening of the La distribution close to the interface was observed in the simulation. This can be attributed to the high swapping probability of La with other atoms seen in the simulations. This happens especially often with Y (see Figure S15 in the Supporting Information), which leads to a distribution of La atoms beyond the inter-diffusion region. As the employed MC approach samples thermodynamic probabilities, it is likely that the diffusion of large La cations into the regular oxygen lattice of YSZ is kinetically hindered and thus not seen as dominantly in the experiment.

The widths given in Figure $4 \mathrm{c}$ are represented by $\sigma$, the standard deviation of the normal cumulative distribution function used to fit the elemental profiles. Some additional long range diffusion of Mn into the YSZ region up to $35 \mathrm{~nm}$ was observed at different LSM/YSZ interfaces (Figure S16 in the Supporting Information), explaining the residual signal in the experimental profile in Figure 4d. Together with the structural characterization results in the next sections, the recurring compositional variations and matching widths at different locations (see Figure S17 in the Supporting Information) indicate that this distinct interfacial region can be classified as a complexion. Complexions are characterized by e.g. structural and compositional 
gradients, partial amorphization and thermodynamic self-limitation. ${ }^{24]}$

However, not all elements are present in this complexion: a systematic shift of $0.8 \mathrm{~nm}$ in the Sr signal (pink line) with respect to the other elements was found in the experimental as well as the simulated distributions. Consequently, it is the only element not contributing to the intergranular region, additionally acting as proof that the observed inter-diffusion is not the result of a sample preparation artifact since this would be expected to lead to a uniform distribution of all elements. The absence of Sr at the YSZ/LSM interface has been reported before for a lower Sr doping. ${ }^{[13]}$ However, no explanation for this observation could be given so far. The swapping probability as obtained by MC (compare Figure S15 in the Supporting Information) unveils that $\mathrm{Sr}$, the largest cation within both oxides, ${ }^{[32]}$ preferentially occupies large cuboctahedral sites (A-sites in the perovskite structure) of LSM. This suggests that, in contrast to the perovskite, a breakdown of large-sized anion polyhedra occurs at the inter-diffusion region, effectively forming a barrier for Sr that leads to a not fully stochastic distribution of ions.

This is supported by a more detailed analysis of the interfacial region, which indeed indicates a change of the cation coordination number in the complexion (compare Figure S18 in the Supporting Information). The reason for this is presumably the deformation of the $\left[\mathrm{ZrO}_{8}\right]$ oxygen cubes in YSZ to octahedra via a bicapped antiprism, as demonstrated in Figure S19 in the Supporting Information. This goes hand in hand with a decrease of the number of neighboring polyhedra for all cation environments and a change of the polyhedron connections (compare Figure S20 and Figure S21 in the Supporting Information). The structure of the complexion is analyzed in further detail in the following.

\subsubsection{Structure of the YSZ/LSM Interface}

The complexion was further investigated structurally by atomically resolved HR-STEM. The high-resolution BF-STEM micrograph (Figure 5a, refer to Figure S23 of the Supporting Information for the corresponding HAADF-STEM image) displays the same YSZ/LSM electrolyte/electrode grain boundary that was investigated by STEM-EDX in Figure 4. YSZ is oriented along the [110] zone axis, as evidenced by the diamond-shaped distribution of $\mathrm{Zr}$ atoms. The corresponding projection of the YSZ crystal structure is presented in Figure S3 in the Supporting Information. The image is oriented such that the [001] axis is horizontal and the [1]̄0] orientation points vertically towards the interface. A more detailed analysis is given in Figure S24 in the Supporting information, where the inset shows the template-matching- 


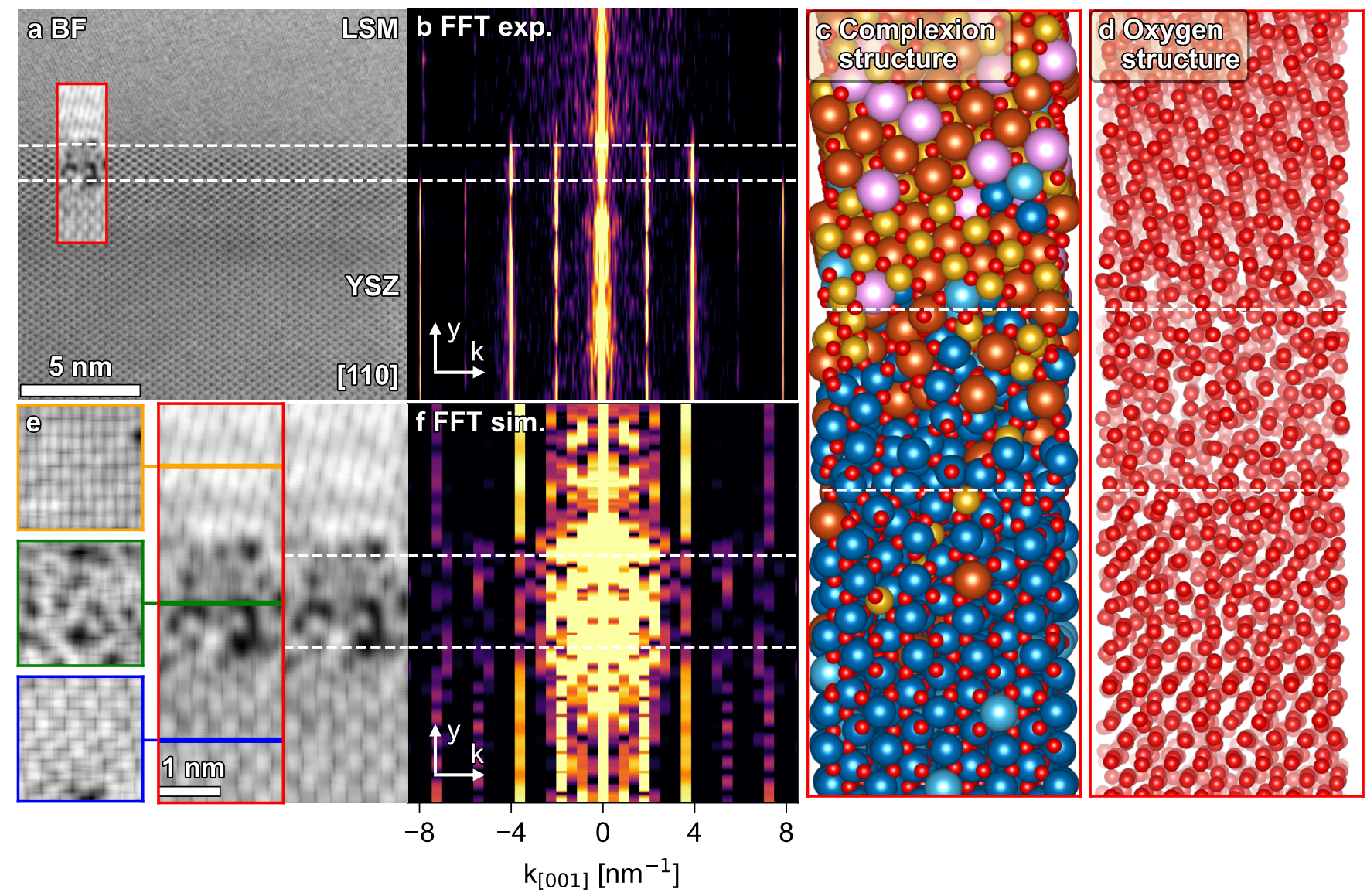

Figure 5. a) High-resolution BF-STEM image of the YSZ/LSM grain boundary with a superimposed cation density from the MC simulation (inset) in [110] zone axis. The white dashed lines enclose the area that is considered to belong to the complexion, based on the analysis in Figure S22 in the Supporting Information. b) Semi-reciprocal plot, i.e. the spatially resolved Fourier Transform along the [001] direction (the $y$ axis corresponds to the real space [110] axis of the micrographs and the $x$ axis is shared with (f). c) View of the inset of (a) at atomic scale with La (bronze), Sr (pink), Mn (yellow), Zr (dark blue), Y (light blue) and O (red). d) Oxygen ions of the structure shown in c. e) Cation density of the simulated interface (right, enlarged version of the inset in (a) and one repetition to show periodicity) with cuts through LSM (left, orange), the complexion (green) and YSZ (blue) that are orthogonal to the paper plane (and parallel to the interface plane). f) Corresponding Fourier Transform of the simulated 3D density with a representation analogous to b.

assisted average of several YSZ unit cells. The analysis clearly reveals the locations of O sites and the missing distortion of the oxygen lattice that would be characteristic for tetragonal zirconia. ${ }^{[31,33]}$ This further corroborates the cubic structure as determined by XRD measure- 
ments (Figure S1c). LSM appears in a high-index zone axis ([135], Figure 5a). Distinct atomic columns are absent, but lattice fringes attributed to $\{310\}$ and $\{2 \overline{1} 1\}$ planes can be observed.

In Figure 5b, spatially resolved 1D Fast Fourier Transforms (FFTs) along the [001] direction of YSZ are plotted, which were obtained from the BF-STEM image that is presented in Figure 5a (see Methods section for more details). While the $x$ axis is in Fourier space ( $k$, sampled along the [001] direction), the $y$ axis of the FFT plot describes real space and is shared with the adjacent HR-STEM image. This semi-reciprocal plot shows strong signals in the YSZ phase (Figure 5b, bottom) and weaker reflections in the LSM phase (Figure 5b, top) due to the less resolved features owing to the high-index zone axis. However, approaching the YSZ/LSM grain boundary, the YSZ signals at high $k$ values (about 6 and $8 \mathrm{~nm}^{-1}$ ) disappear (Figure 5b, middle, dashed white lines), and only the peaks at 2 and $4 \mathrm{~nm}^{-1}$ remain, as also evident from the line profiles presented in Figure S22 in the Supporting Information. The loss of signals at high $k$ can be understood as the loss of high index reflections due to reduction of long range order in the diffusion layer. As low index reflections at low $k$ remain, short range order is still present. Thus, this points toward the formation of a less ordered/slightly amorphous structure on the YSZ side of the grain boundary.

Figure 5c shows the resulting grain boundary geometry according to the MC simulations. While LSM (top) and YSZ (bottom) both possess clear crystalline order, the diffusion layer appears more disordered and forms a structurally and compositionally distinct region between the two materials, denoted as complexion. ${ }^{[21]}$ The loss of translational symmetry within the complexion is also evident when observing only the oxygen ions in the structure as they define the boundaries of the involved crystal structure. This is shown in Figure 5d. In order to analyze the amorphization of this layer, the corresponding cation density is mimicked by $3 \mathrm{D}$ Gaussian functions, whose 2D projection is given in Figure 5e (right). Additionally, crosssectional cuts through the cation densities of LSM (yellow), YSZ (blue) and the complexion (green) are shown to provide a complete picture of the degree of crystallinity in all three dimensions. The corresponding windowed FFT of the 3D density of the simulated cell (f) is in good agreement with the experimental FFT. For YSZ (Figure 5e, bottom), the position of the signals agree with the experimental ones, although they are slightly lower as a result of the marginally stretched cell size needed for compliance with LSM. The peaks in the upper half of the graph result from LSM with a [135] surface termination. However, the LSM grain boundary orientation seems to be different from the experiment, as the few peaks that can be spotted 
are shifted. Nevertheless, the simulated FFT of the complexion qualitatively agrees with the experiment, indicating that the LSM interface orientation is not crucial for the characteristics of the formed complexion. Like in the experimentally determined FFT, signals at high $k$ vanish at the complexion in Figure 5f, affirming that long range order is lost, while the signals at low $k$ from YSZ persists, which ascertains that some structure is retained for short to medium distances. Hence, the cations of the formed complexion preserve the short range structure of the YSZ phase, which can distinctly be seen in the BF-STEM image (Figure 5a). The loss of long range order derives from the disturbance of the oxygen ion lattice by the doping ions $\mathrm{Mn}$ and La, such that, in the complexion, many anions can be found aside defined crystal positions. This uneven framework of the oxygen atoms in turn causes distortion of the cation positions, leading to a slight amorphization of the phase and the loss of large cation sites which are typical for the perovskite-structured LSM.

\subsubsection{Complexion Classification}

Earlier reports did not detect this transitional region between cubic 8YSZ and LSM; however, a zone of cubic YSZ has been described for the interface between the otherwise tetragonal 3 YSZ (i.e. YSZ with 3 mol\% of yttria) and LSM. ${ }^{10,13]}$ Therein, this region was interpreted as a nanometer-wide solid solution with strong lattice distortion on the YSZ side of the interface with particular chemistry. The suggested formation of solid solutions, ${ }^{[13]}$ for which $\mathrm{Y}^{3+}, \mathrm{La}^{3+}$ or Mn cations can be homogeneously dispersed in zirconia, should only lead to a slight relaxation of atomic positions, whereas the complexion observed here undergoes such a strong deformation that crystallographic reflections are lost. This is especially pronounced in the oxygen positions in Figure 5d. The high degree of disorder caused by the diffusion-induced doping and the resulting loss of long range order suggests that this cannot be classified as a solid solution. On the other hand, it also cannot be referred to as an interphase since, based on the amporphization of the oxygen lattice in the inter-diffusion layer, thermodynamic stability as an extended 3D phase without the confining bulk phases is highly unlikely. Additionally, the stoichiometric variations introduced by the cross-diffusion do not satisfy the definition of the term 'phase'. However, in addition to other properties discussed in the following, partial amorphization and compositional gradients are characteristic features of complexions. ${ }^{[23,24]}$ Thus, in contrast to the previously proposed models of the YSZ/LSM interface, the results presented here, for the first time, reveal that considering the concept of complexion formation is necessary as it does not 
only explain the general structural and compositional appearance, but also the strong variation of the chemical properties at the YSZ/LSM boundary compared to the respective bulk phases that have also been observed for similar interfaces. ${ }^{[25]}$

The investigated complexion is characterized by compositional and structural profiles which deviate from either of the neighboring LSM and YSZ phases distinctively. Structural changes are necessitated by the fact that the anion is the mobile species in the less rigid solid ion conductor YSZ, which forms a contact with the robust, perovskite-structured and electronically conducting LSM by structurally adjusting over a finite width. The thermodynamic driving force for this might be seen in a frustrated phase transformation where the complexion resembles a kinetically trapped intermediate or precursor state on the way to the formation of the undesired pyrochlore $\mathrm{La}_{2} \mathrm{Zr}_{2} \mathrm{O}_{7}$, which is known to be formed if the material is sintered at too high temperatures. ${ }^{[34-36]}$ Alternatively, it is also known that LSM is less prone to pyrochlore formation than, for example, cobalt- and iron-based perovskites, ${ }^{[37]}$ and exhibits a higher electrocatalytic stability than those materials. ${ }^{[38]}$ Both of these properties might have their origin in the presence of this complexion.

The width of a complexion is self-limited and dependent on various intrinsic and extrinsic factors such as surface orientation, temperature and pressure. As evident from analysis of the disordered region in Figure S22 in the Supporting Information, the observed complexion spans approximately four atomic layers $(1.47 \mathrm{~nm})$, which classifies as multilayer in Dillon-Harmer nomenclature. ${ }^{[21]}$ However, in contrast to the strongly amorphous multi-layer complexions in alumina, the retained structural order in YSZ/LSM corresponds more to the bi- or trilayer structures, illustrating that complexion characteristics are very material-specific.

In all experimentally probed sample regions, the thicknesses of all grain boundaries are similar (compare Figure S17 in the Supporting Information). This is in agreement with the thermodynamic control of complexion formation, whereas, in the kinetic regime of a non-self-limited layer, a significantly higher variety of complexion thicknesses would be expected. However, the thermodynamic stability of the complexion under reaction conditions remains unclear as defects, such as the distortions of the anionic lattice shown in Figure 5 can act as kinetic barriers as well. On the other hand, the thermodynamical self-limited width is also observed in the simulations (see Figure 4 and Figure S25 in the Supporting Information for different interface terminations), where complexion growth is limited in the approached thermodynamic minimum. Comparing the two simulations, the sensitivity of complexion properties such as width and degree of amorphization on the confining bulk lattice orientation can also be observed. 
The dependence of the form of complexions on multiple parameters implies that their properties correlate with various conditions that can be controlled experimentally. Knowing the relationship between relevant complexion features and adjustable parameters thus allows the selective tuning of relevant complexion properties such that a beneficial influence of the complexion on the active phase activity can be achieved. Identifying these correlations and connecting the impact of the complexion characteristics to the TPB under working conditions requires a detailed operando study, designated to link complexion properties to cell performance. With the aid of theoretical models, a further link to intrinsic characteristics of the different complexion categories such as ionic and electronic conductivity can also be established. This ultimately compiles to the creation of a YSZ/LSM complexion phase diagram and property map that enables finding the optimal operation conditions to enhance the performance and lifetime of SOCs.

Beyond the questions whether the YSZ/LSM complexion constitutes a mixed ionic/electronic conductor that can supply the reactive centers at the surface of the TPB with the charged reactants from 'underneath', it is of paramount importance to establish the structure of the living catalytic TPB surface under operating conditions. A legitimate path would be to follow the general assumption of surface science that the native and active catalyst surface is derived from the underlying structure of the bulk. In the present case, it would be expressed by the structure of the YSZ/LSM complexion. However, for metal oxides, it is known that the surface structure can differ substantially from the underlying bulk. ${ }^{[39-41]}$ Further modifications are induced in the surface and sub-surface layers due to varying chemical potentials of the gas phase species that are in contact with the outermost layer of this complexion at elevated temperatures. As the electric and ionic conductivity of the complexion remains unknown, it will be important to know how this two-dimensional surface termination contributes to the appearance and function of the expected one-dimensional TPB.

These intricate new questions of structural and compositional variability along two orthogonal dimensions, i.e. vertical solid/gas and lateral YSZ/LSM, require spatially resolved operando experiments. For operando TEM experiments, atomic resolution is possible, ${ }^{[42]}$ although a spatially resolved SOEC/SOFC operando investigation would require new cell developments that allow for realistic measurements. Even so, it remains questionable whether the observed atomic description of the surface structure that may be observed under operando conditions is relevant or significant. For instance, high-resolution imaging requires a high electron density. 
For operando experiments, it is, thus, impossible to disentangle between reaction-, electron beam-, and radical-induced surface structures, i.e. when the electron beam interacts with the gas phase. ${ }^{43]}$ This example shows that spatially resolved operando techniques alone may be of limited use and should be complemented by quasi in situ imaging approaches and theory. ${ }^{[44,45]}$

\section{Conclusion}

By unraveling a complexion structure at the electrolyte/electrode interface of SOECs/SOFCs at the atomic scale, we have paved the way to a more realistic picture of solid oxide cells and their functionality. Driven by significant inter-diffusion of all cations except for Sr, a breakdown of the oxygen lattice leads to the formation of this complexion, i.e. a thermodynamically stable, nanometer-sized intergranular layer of reduced long-range order, which was observed by experiment and theory.

The inclusion of finite structures such as complexions in the classically simplified model of an atomically sharp interface is an important extension with immediate impact on the appearance and performance of the active surface area. We also expect it to be a powerful concept for chemical interface engineering toward more active and long-lived cells.

\section{Supporting information}

Additional characterization by XRD, supplementary STEM images and EDX spectrum images, EELS data and calculations, calculation details and additional analyses of the complexion.

\section{Author contributions}

H.T., F.-P.S. and T.G. have performed the main experiments/calculations and their analysis and have written the majority of the manuscript. F.G. has conducted the XRD experiments and analyses and contributed to the crystallographic discussions. A.H. and D.I. have prepared the TEM specimens and have acquired and interpreted the SEM images. I.C.V. and L.G.J.d.H. designed the electrodes and provided expertise. A.K.-G., C.S. and T.L. have supervised the experiments/simulations and discussions and have contributed in writing the manuscript. R.A.E., K.R. and R.S. provided perspectives and acquired funding. 


\section{Conflicts of interest}

There are no conflicts of interest to declare.

\section{Acknowledgements}

This work was supported by the Deutsche Forschungsgemeinschaft (DFG, German Research Foundation) under the priority programme SPP 2080 DynaKat. T. Götsch additionally acknowledges funding by the Fonds zur Förderung der wissenschaftlichen Forschung (FWF, Austrian Science Fund) via project number J4278. F.-P. Schmidt acknowledges funding by the Deutsche Forschungsgemeinschaft (DFG, German Research Foundation) - 388390466 TRR 247. Moreover, this work was partially funded by the Deutsche Forschungsgemeinschaft (DFG, German Research Foundation) under Germany's Excellence Strategy — EXC 2089/1 — 390776260. The authors acknowledge the Helmholtz-Zentrum Berlin für Materialien und Energie for allocating beam time within proposal number 201-09080CR. Additionally, the authors thank V. Vibhu for preparation of the electrodes.

\section{Keywords}

Electron Microscopy, Fuel Cells, Interfaces, Molecular Modeling

\section{References}

[1] M. Ni, M. Leung, D. Leung, Int. J. Hydrogen Energy 2008, 33, 2337-2354.

[2] Q. Cai, C. S. Adjiman, N. P. Brandon, J. Power Sources 2014, 268, 212-224.

[3] R. M. Ormerod, Chem. Soc. Rev. 2003, 32, 17-28.

[4] A. J. Jacobson, Chem. Mater. 2010, 22, 660-674.

[5] A. Atkinson, S. Barnett, R. J. Gorte, Nat. Mater. 2004, 3, 17-27.

[6] M. S. Sohal, J. E. O’Brien, C. M. Stoots, V. I. Sharma, B. Yildiz, A. Virkar, J. Fuel Cell Sci. Tech. 2012, 9, 011017.

[7] K. Chen, S. P. Jiang, J. Electrochem. Soc. 2016, 163, F3070. 
[8] M. Tahir, L. Pan, F. Idrees, X. Zhang, L. Wang, J.-J. Zou, Z. L. Wang, Nano Energy 2017, 37, 136-157.

[9] L. Han, S. Dong, E. Wang, Adv. Mater. 2016, 28, 9266-9291.

[10] M. Backhaus-Ricoult, M. Badding, J. Brown, M. Carson, E. Sanford, Y. Thiaul, Developments in solid oxide fuel cells lithium ion batteries 2005, 21-30.

[11] M. Keane, M. K. Mahapatra, A. Verma, P. Singh, Int. J. Hydrogen Energy 2006, 37, $16776-16785$.

[12] M. C. Brant, T. Matencio, L. Dessemond, R. Z. Domingues, Solid State Ion. 2006, 177, 915-921.

[13] M. Backhaus-Ricoult, Solid State Ionics 2006, 177, 2195-2200.

[14] M. Backhaus-Ricoult, K. Adib, T. St.Clair, B. Luerssen, L. Gregoratti, A. Barinov, Solid State Ionics 2008, 179, 891-895.

[15] Y. L. Liu, A. Hagen, R. Barfod, M. Chen, H. J. Wang, F. W. Poulsen, P. V. Hendriksen, Solid State Ion. 2009, 180, 1298-1304.

[16] S. N. Rashkeev, M. V. Glazoff, Int. J. Hydrogen Energy 2012, 37, 1280-1291.

[17] S. He, S. P. Jiang, Prog. Nat. Sci. Mater. Int. 2021, doi: 10.1016/j.pnsc.2021.03.002.

[18] J. A. Kilner, R. A. De Souza, I. C. Fullarton, Solid State Ionics 1996, 86-88, 703-709.

[19] P. S. Manning, J. D. Sirman, J. A. Kilner, Solid State Ionics 1997, 93, 125-132.

[20] S. B. Adler, X. Y. Chen, J. R. Wilson, J. Catal. 2007, 245, 91-109.

[21] S. J. Dillon, M. Tang, W. C. Carter, M. P. Harmer, Acta Mater. 2007, 55, 6208-6218.

[22] J. Luo, Crit. Rev. Solid State Mater. Sci. 2007, 32, 67-109.

[23] P. R. Cantwell, M. Tang, S. J. Dillon, J. Luo, G. S. Rohrer, M. P. Harmer, Acta Mater. 2014, 62, 1-48.

[24] J. Luo, Energy Storage Mater. 2019, 21, 50-60. 
[25] J. Timmermann, F. Kraushofer, N. Resch, P. Li, Y. Wang, Z. Mao, M. Riva, Y. Lee, C. Staacke, M. Schmid, C. Scheurer, G. S. Parkinson, U. Diebold, K. Reuter, Phys. Rev. Lett. 2020, 125, 206101.

[26] J. Rodríguez-Carvajal, M. Hennion, F. Moussa, A. H. Moudden, L. Pinsard, A. Revcolevschi, Phys. Rev. B 1998, 57, R3189-R3192.

[27] X. Qiu, T. Proffen, J. F. Mitchell, S. J. Billinge, Phys. Rev. Lett. 2005, 94, 1-4.

[28] T. Grande, J. R. Tolchard, S. M. Selbach, Chem. Mater. 2012, 24, 338-345.

[29] R. J. D. Tilley, Perovskites: Structure-Property Relationships, John Wiley \& Sons, Ltd, Chichester, UK, 2016.

[30] T. Götsch, L. Schlicker, M. F. Bekheet, A. Doran, M. Grünbacher, C. Praty, M. Tada, H. Matsui, N. Ishiguro, A. Gurlo, B. Klötzer, S. Penner, RSC Adv. 2018, 8, 3120-3131.

[31] H. Horiuchi, A. J. Schultz, P. C. Leung, J. M. Williams, Acta Crystallogr. Sect. B 1984, 40, 367-372.

[32] R. D. Shannon, Acta Cryst 1976, A32, 751-767.

[33] B. Bondars, G. Heidemane, J. Grabis, K. Laschke, H. Boysen, J. Schneider, F. Frey, J. Mater. Sci. 1995, 30, 1621-1625.

[34] K. Yang, J. H. Shen, K. Y. Yang, I. M. Hung, K. Z. Fung, M. C. Wang, J. Power Sources 2006, 159, 63-67.

[35] J. A. Labrincha, J. R. Frade, F. M. Marques, J. Mater. Sci. 1993, 28, 3809.

[36] A. Chen, J. R. Smith, K. L. Duncan, R. T. DeHoff, K. S. Jones, E. D. Wachsman, J. Electrochem. Soc. 2010, 157, B1624.

[37] W. Wang, Y. Huang, S. Jung, J. M. Vohs, R. J. Gorte, J. Electrochem. Soc. 2006, 153, A2066.

[38] A. Neumann, M. N. H., I. Vinke, H. Lippert, ECS Trans. 2009, 25, 2889-2898.

[39] L. Masliuk, F.-P. Schmidt, W. Hetaba, M. Plodinec, G. Auffermann, K. Hermann, D. Teschner, F. Girgsdies, A. Trunschke, R. Schlögl, T. Lunkenbein, J. Phys. Chem. C 2020, 124, 23069-23077. 
[40] X. Li, T. Lunkenbein, V. Pfeifer, M. Jastak, P. K. Nielsen, F. Girgsdies, A. Knop-Gericke, F. Rosowski, R. Schlögl, A. Trunschke, Angew. Chemie Int. Ed. 2016, 55, 4092-4096.

[41] K. Reuter, Catal. Letters 2016, 146, 541-563.

[42] M. Plodinec, H. C. Nerl, F. Girgsdies, R. Schlögl, T. Lunkenbein, ACS Catal. 2020, 10, 3183-3193.

[43] M. Boniface, M. Plodinec, R. Schlögl, T. Lunkenbein, Top. Catal. 2020, 63, 1623-1643.

[44] L. Masliuk, M. Swoboda, G. Algara-Siller, R. Schlögl, T. Lunkenbein, Ultramicroscopy 2018, 195, 121-128.

[45] S. Janbroers, J. N. Louwen, H. W. Zandbergen, P. J. Kooyman, J. Catal. 2009, 268, 235-242. 